\title{
Diferencias de las condiciones mecánicas de un suelo arcilloso sometido a diferentes sistemas de labranza
}

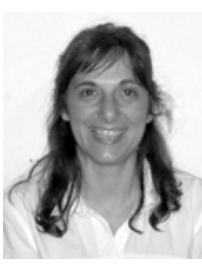

\author{
Laura M. Draghi ${ }^{1}$, Guido F. Botta ${ }^{1}$, Roberto H. Balbuena ${ }^{1}$, Jorge A. Claverie ${ }^{1}$ \& Héctor Rosatto ${ }^{2}$ \\ 1 Dto. de Ingeniería Agrícola y Forestal, Facultad de Cs. Agrarias y Forestales de la UNLP. Avenida 60 y 119 , CP 1900 , \\ La Plata, Argentina. E-mail: Idraghi@ceres.agro.unlp.edu.ar (Foto) \\ 2 Facultad de Agronomía de la UBA. Avda San Martín 4453, CP 1417, Buenos Aires, Argentina. E-mail: sad@s6coopenet.com.ar
}

Protocolo 71 - 20/5/2002 - Aprovado em 1/3/2004

\begin{abstract}
Resumen: El objetivo del presente trabajo fue cuantificar la reacción mecánica del suelo al tránsito, a través de la resistencia a la penetración y la densidad aparente para visualizar posibles diferencias debido al sistema de labranza utilizado. El ensayo se instaló sobre un suelo Argiudol típico, sobre dos lotes, provenientes cada uno de seis años de cultivo trigo-soja bajo dos formas de labranza: siembra directa (SD) y labranza convencional (LC). Se establecieron 4 tratamientos de tránsito, correspondientes a $6,8,10$ y 12 pasadas de un tractor de diseño convencional (2WD) Massey Fergusson 1175 de 52,25 kW (71 CV) en el motor. Para determinar los efectos del tránsito sobre la compactación inducida, se determinaron la densidad global (DA) y la resistencia a la penetración (RP). Luego de 6 años de rotación trigo-soja bajo estas dos formas de cultivo (siembra directa y labranza convencional) la condición mecánica de los suelos resultó ser significativamente diferente, al menos en las capas más superficiales, resultando el suelo trabajado con SD mayores valores de RP que la condición de LC. La siembra directa registró valores de DA limitantes para el normal desarrollo radicular a menores intensidades de tráfico y desde menores profundidades. A medida que aumentó la intensidad de tráfico disminuyó la profundidad donde se alcanzaron valores de resistencia a la penetración potencialmente determinantes de la detención en el crecimiento radicular.
\end{abstract}

Palabras clave: compactación inducida, siembra directa, resistencia a la penetración

\section{Differences in mechanical conditions of a clayey soil under different tillage systems}

\begin{abstract}
With the aim to evaluate the soil mechanic reaction-traffic relationship to different tillage systems, penetration resistance and soil bulk density measurements were made. Four traffic conditions $(6,8,10$ and 12 passes of a $2 W D, 52.25 \mathrm{~kW}$ tractor) were evaluated in a typic Argiudol soil with six years of wheat-soybean rotation under no-tillage and conventional tillage. Bulk density and penetration resistance were used to evaluate the traffic effects on soil compaction. After the six-year rotation, no-tillage system showed significant higher penetration resistance values with respect to conventional tillage system in the topsoil layer. The bulk density values recorded in the non tillage system determine root growth limitation at low number of passes and at shallow depth. The greater the number of passes, lower the depth at which the penetration resistance can reach potential root growth limiting values.
\end{abstract}

Key words: induced compaction, no tillage, penetration resistance

\section{INTRODUCCIÓN}

La siembra directa es, sin duda, un sistema muy utilizado en la producción agrícola moderna de la Argentina, existiendo en la actualidad más de 10 millones de hectáreas (Consejo Federal de Inversiones, 2001) cultivadas según esta modalidad, contribuyendo a su muy rápida difusión la técnica del doble cultivo trigo-soja. Si bien la concepción del sistema tiende a mejorar la conservación del recurso suelo, coexisten resultados contradictorios en cuanto a la compactación inicial de los suelos, como así también de su evolución y persistencia en el tiempo. Algunos investigadores no han encontrado efectos significativos de diferentes sistemas de labranza en la compactación de los suelos (Ismail et al. 1995) y en su 
distribución vertical (Flowers \& Lal, 1998). Por otro lado, Domínguez et al. (2000) han registrado una mayor acumulación de compactación superficial y una mayor capacidad portante en suelos bajo siembra directa. En el mismo sentido, Carter (1988) informó que los sistemas que no remueven el suelo pueden ayudar a la compactación del mismo.

La resistencia a la penetración (RP) y la densidad global (DA) son dos parámetros que usualmente se utilizan para cuantificar compactaciones producto del tráfico vehicular. Daddow \& Warrington (1983) establecieron que $1,65 \mathrm{Mg} \mathrm{m}^{-3}$ es el umbral de densidad aparente para permitir el crecimiento radicular. Numerosos trabajos han establecido rangos de resistencia a la penetración que serían responsables de causar restricciones para el normal desarrollo radicular. Bowen et al. (1994) citan valores entre 0.9 a 1.5 MPa como límites para impedir el crecimiento satisfactorio de las raíces. Threadgill (1982) fija $1.5 \mathrm{MPa}$ y $2.5 \mathrm{MPa}$ como valores responsables del desarrollo anormal y de la detención del desarrollo radicular para el caso de árboles frutales.

Jorajuría \& Draghi (2000), en relación con el pasaje repetido de tractores de baja carga/eje, concluyen que el pasaje repetido sobre la misma senda puede emular e incluso reemplazar al factor peso sobre el eje, en la responsabilidad principal de inducir compactaciones en el subsuelo. Jorajuría (2001) indicó, además, que la profundidad del horizonte del suelo que reacciona con un mayor incremento de la resistencia a la penetración tiende a hacerse más superficial, en la medida que aumenta el número de pasadas. Botta (2000) concluye que, en forma independiente del peso y de la superficie de contacto rueda/ suelo, el número de pasadas reiteradas en una misma senda inducen compactación subsuperficial del suelo. En relación a los efectos de la presión en el área de contacto rueda/ suelo, van den Akker (1998) encontró que la inducción de compactaciones por tráfico, con $32 \mathrm{kN}$ en el eje, puede mantenerse dentro del horizonte arable (Ap), utilizando un rodado que garantice una mayor superficie de contacto rueda/ suelo y una menor presión de inflado. Alakukku (1997) recomendó que el tránsito debiera reducirse al mínimo indispensable en condiciones de elevada humedad y los vehículos debieran portar ruedas que les permitieran limitar su presión de inflado a una máxima de $50 \mathrm{kPa}$. Las consecuencias, de no lograr establecer estas soluciones, adquieren mayor relevancia, cuando las condiciones agroclimáticas resultan desfavorables.

El objetivo del presente trabajo fue cuantificar la reacción mecánica del suelo al tránsito, a través de la resistencia a la penetración y la densidad aparente, para visualizar posibles diferencias debido al sistema de labranza utilizado.

\section{MATERIAL Y MÉTODOS}

El ensayo se instaló sobre un suelo Argiudol típico, de la serie Río Tala, ubicado en el partido de Baradero, provincia de Buenos Aires, Argentina. Se trabajó sobre un lote en producción con seis años de cultivo trigo-soja bajo dos formas de labranza: siembra directa (SD) y labranza convencional (LC). Las determinaciones se realizaron en el mes de enero sobre restos de rastrojo de trigo y soja ya implantada.
Desde el año 1995 las labores utilizadas en el sistema de laboreo convencional fueron una combinación de un pasaje de rastra de discos semipesada ( $70 \mathrm{~kg} /$ disco), laboreo con escarificador de cinceles y rastra de discos, nuevamente, para preparación de la cama de siembra. En el sistema de siembra directa se realizó un único pasaje de la máquina sembradora sin roturación previa del suelo. Las operaciones de cosecha se realizaron con tránsito de la cosechadora y los carros graneleros desplazándose en forma conjunta por el predio para la descarga del cereal. En función de lo expuesto, y contabilizando la aplicación eventual de funguicidas e insecticidas para ambos cultivos, se estimó un número mínimo de 6 pasadas y un número máximo entre 8 y 12 pasadas de vehículos sobre el terreno. Se establecieron por lo tanto 4 tratamientos de tránsito, correspondientes a $6,8,10$ y 12 pasadas del tractor, todas en un mismo día para garantizar uniformidad en las condiciones que tienen decisivo efecto sobre las variables cuantificadas. El diseño experimental utilizado fue en bloques completamente al azar, con tres repeticiones, dentro de los cuales se ubicaron los distintos tratamientos de tránsito, los cuales se contrastaron contra un testigo sin tráfico de vehículos. Las parcelas experimentales tuvieron una longitud de $50 \mathrm{~m}$, dentro de las cuales se establecieron las parcelas de medición, de $30 \mathrm{~m}$ de longitud, en el sector medio de las mismas.

Se utilizó un tractor de diseño convencional (2WD) Massey Fergusson 1175 de 52,25 kW (71 CV) en el motor. Masa del vehículo: $3,02 \mathrm{Mg}$, eje delantero: $0,94 \mathrm{Mg}$ eje motriz: $2,08 \mathrm{Mg}$ Rodado trasero: 16.9-34. Rodado delantero: 7.50-16. Las presiones en el área de contacto rueda suelo resultaron de 142,65 y $110,55 \mathrm{kPa}$ para los rodados delanteros y traseros respectivamente.

\section{Del suelo}

El perfil del suelo posee un horizonte $A_{11}$ de $0,16 \mathrm{~m}$ de desarrollo, franco limoso, con $261 \mathrm{~g} \mathrm{~kg}^{-1} \mathrm{de}$ arcilla, $568 \mathrm{~g} \mathrm{~kg}^{-1} \mathrm{de}$ limo y $171 \mathrm{~g} \mathrm{~kg}^{-1} \mathrm{de}$ arena. El horizonte $\mathrm{A}_{12}$ entre 0,16 y $0,30 \mathrm{~m} \mathrm{de}$ profundidad es de textura franco arcillo limosa, con $350 \mathrm{~g} \mathrm{~kg}^{-1}$ de arcilla, $500 \mathrm{~g} \mathrm{~kg}^{-1}$ de limo y $150 \mathrm{~g} \mathrm{~kg}^{-1}$ de arena. A partir de los $0,30 \mathrm{~m}$ y hasta los $0,50 \mathrm{~m}$ se desarrolla un horizonte $B_{21,}$, arcilloso, estructura en prismas compuestos regulares gruesos fuertes, extremadamente duro en seco, firme en húmedo, muy plástico y adhesivo. El contenido de arcilla es de $508 \mathrm{~g} \mathrm{~kg}^{-1}, 392 \mathrm{~g} \mathrm{~kg}^{-1}$ de limo y $100 \mathrm{~g} \mathrm{~kg}^{-1}$ de arena. A continuación se desarrolla hasta $0,84 \mathrm{~m}$ un horizonte $\mathrm{B}_{22 \mathrm{t}}$, arcillo limoso, estructura en prismas compuestos regulares gruesos fuertes, extremadamente duro en seco, muy firme en húmedo, plástico y adhesivo (493 $\mathrm{g} \mathrm{kg}^{-1}$ de arcilla, $413 \mathrm{~g} \mathrm{~kg}^{-1}$ de limo y $94 \mathrm{~g} \mathrm{~kg}^{-1}$ de arena). El contenido de materia orgánica para los horizontes descriptos fue del 3,36, 2,64, 1,50 y $1,15 \%$ respectivamente.

\section{De las variables experimentales.}

Para determinar los efectos del tránsito sobre la compactación inducida se determinaron la densidad aparente y la resistencia a la penetración. Además se registró la humedad gravimétrica del suelo, mediante un calador cada $15 \mathrm{~cm}$ de profundidad.

La densidad global se midió por medio de cilindros de volumen conocido en tres intervalos de profundidad: 0 a 15 
$\mathrm{cm}, 15-30 \mathrm{~cm}$ y $30-60 \mathrm{~cm}$. Se realizaron 3 repeticiones de cada dato en cada parcela de medición.

Para la resistencia a la penetración se utilizó un penetrómetro Agridry Rimik, modelo CP 20 y metodología según ASAE S 313.4 (1992) hasta una profundidad de $57,5 \mathrm{~cm}$ cada $2,5 \mathrm{~cm}$. Se realizaron 15 repeticiones de cada dato, en cada parcela experimental.

Sobre los datos se realizó un análisis de la varianza, cuantificándose la significancia de las diferencias por medio del Test de Tukey $(\mathrm{p} \leq 0,05)$.

\section{RESULTADOS Y DISCUSIÓN}

En la Tabla 1 se visualizan los valores de humedad de los distintos estratos analizados.

Tabla 1. Valores de humedad (\%) para los distintos estratos evaluados*

\begin{tabular}{ccc}
\hline Profundidad $(\mathrm{cm})$ & Labranza convencional & Siembra directa \\
\hline $0-15$ & $22 \mathrm{a}$ & $20 \mathrm{a}$ \\
$15-30$ & $23 \mathrm{a}$ & $23 \mathrm{a}$ \\
$30-45$ & $26 \mathrm{a}$ & $24 \mathrm{a}$ \\
$45-60$ & $25 \mathrm{a}$ & $23 \mathrm{a}$ \\
\hline \% Medias seguidas de la misma letra inicial en las columnas no tienen diferencias significativas al \\
5\% de probabilidades del test de Tukey
\end{tabular}

El contenido de humedad no presentó diferencias entre estratos de profundidad analizados y tratamientos estudiados, por lo tanto las diferencias de índice de cono registradas no podrán explicarse por diferencias de este valor.

\section{Condición mecánica de los tratamientos testigos.}

Luego de 6 años de rotación trigo-soja bajo estas dos formas de cultivo (siembra directa y labranza convencional) la condición mecánica del suelo resultó ser significativamente diferente, al menos en las capas más superficiales. Hasta los 20 $\mathrm{cm}$ de profundidad, el suelo que ha recibido siembra directa, presentó mayores valores de RP que la condición de LC, superando en un $80 \%$ en promedio a la misma hasta los $12,5 \mathrm{~cm}$ de profundidad, (Fig. 1) coincidiendo con lo reportado por Domínguez et al. (2000).

A los $20 \mathrm{~cm}$ no se registraron diferencias entre ambas condiciones de suelo. Desde los 22,5 cm y hasta $10 s 37,5 \mathrm{~cm}$ la tendencia se revirtió y fue el suelo que ha recibido labranza convencional, el que presentó valores de resistencia a la penetración significativamente superiores. Sin embargo aquí las diferencias alcanzan en promedio al 10\%. Desde los $40 \mathrm{~cm}$ y hasta los $57,5 \mathrm{~cm}$ la siembra directa nuevamente presentó mayor impedancia mecánica, registrándose valores superiores a 1500 $\mathrm{kPa}$ e incluso superando los $2000 \mathrm{kPa}$ a los 55 y $57,5 \mathrm{~cm}$ de profundidad con lo cual se demuestra que la siembra directa ha generado condiciones que podrían dificultar severamente el crecimiento radicular.

Si se observan los datos de densidad global puede verse que para todas las profundidades analizadas, la densificación registrada en el suelo sometido a siembra directa siempre fue mayor que para el caso de la labranza convencional (Tabla 2).

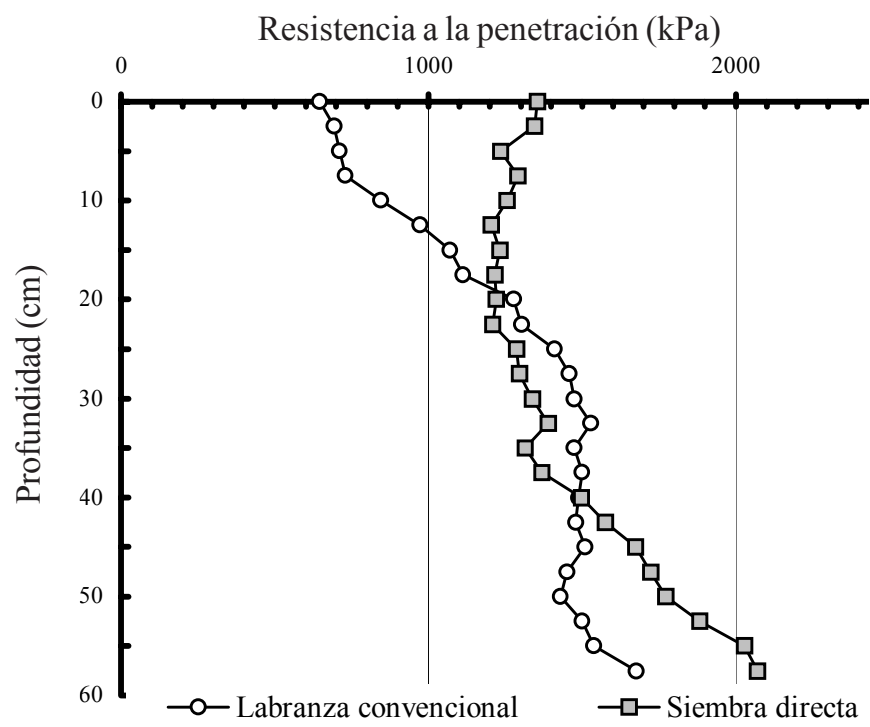

Figura 1. Resistencia a la penetración -RP- $(\mathrm{kPa})$ según las diferentes profundidades $(\mathrm{cm})$ en ambas condiciones de suelos no traficados durante el ensayo

Tabla 2. Valores de densidad global $\left(\mathrm{Mgm}^{-3}\right)$ registrados con cilindros en los suelos testigos ségun las diferentes profundidades $(\mathrm{cm})$

\begin{tabular}{lccc}
\hline & \multicolumn{3}{c}{ Densidad aparente $\left(\mathrm{Mg} \mathrm{m}^{-3}\right)$} \\
\cline { 2 - 4 } & $0-15$ & $15-30$ & $30-60$ \\
\hline Labranza convencional & 1,20 & 1,42 & 1,67 \\
Siembra directa & 1,33 & 1,59 & 1,87 \\
\hline
\end{tabular}

Podría inferirse hasta aquí que la siembra directa ha resultado en una mayor densificación del suelo que la labranza convencional, alcanzándose valores de densidad aparente muy limitantes para el desarrollo óptimo de las raíces desde los 15 $\mathrm{cm}$ de profundidad, superando el umbral citado por Daddow \& Warrington (1983) para el intervalo más profundo.

Podría inferirse hasta aquí que la siembra directa ha resultado en una mayor densificación del suelo que la labranza convencional, alcanzándose valores de densidad aparente muy limitantes para el desarrollo óptimo de las raíces desde los 15 cm de profundidad, superando el umbral citado por Daddow \& Warrington (1983) para el intervalo más profundo.

\section{Incidencia del número de pasadas.}

En el suelo sometido a siembra directa, independientemente de la profundidad analizada, el tratamiento de mayor intensidad de tráfico (12 pasadas) fue el único que aumentó significativamente los valores de resistencia a la penetración de la condición testigo superando $1500 \mathrm{kPa}$ a partir de los 32,5 cm de profundidad (Fig.2).

Además, analizando el aumento de la resistencia a la penetración del suelo testigo para las diferentes intensidades de tráfico y las profundidades alcanzadas, pudo comprobarse que la incidencia del tráfico modificó la condición mecánica del suelo hasta mayores profundidades a medida que aumentó el número de pasadas.

En cuanto a los valores absolutos de resistencia a la penetración iguales ó superiores a $2 \mathrm{MPa}$, aparecieron luego 
de los 55 y $57,5 \mathrm{~cm}$ para el suelo testigo y 6 pasadas, luego de los $52,5 \mathrm{~cm}$ para el tratamiento de 8 pasadas y luego de los 50 $\mathrm{cm}$ de profundidad para el tratamiento de mayor intensidad de tráfico. Se coincide con Jorajuría (2000) en que a medida que aumenta el número de pasadas comienzan a aparecer valores limitantes de RP cada vez en capas de suelo más superficiales.

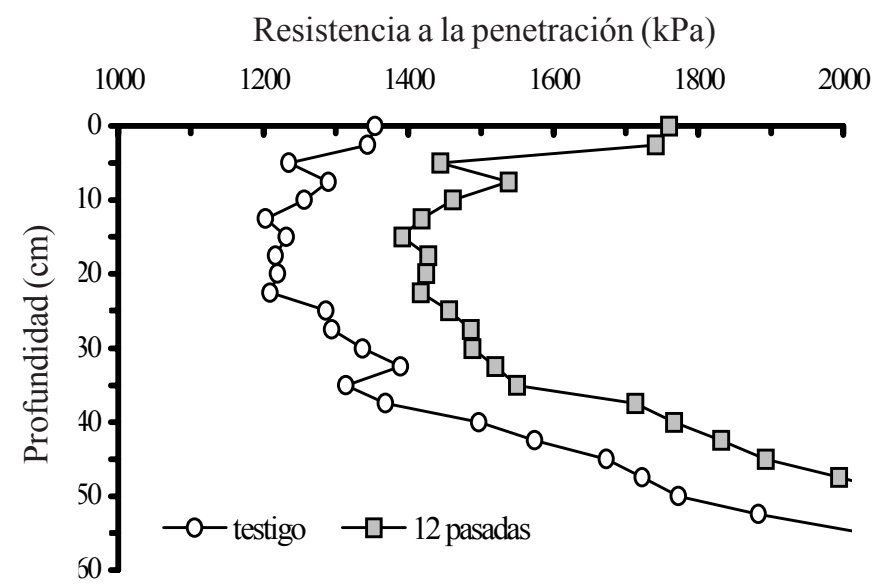

Figura 2. Resistencia a la penetración $(\mathrm{kPa})$ según las diferentes profundidades $(\mathrm{cm})$ en el suelo sometido a siembra directa. Tratamientos testigo y 12 pasadas

Si se analizan los resultados encontrados en el suelo que tiene seis años de labranza convencional, puede verse que para cualquier profundidad, todos los tratamientos aumentaron la resistencia a la penetración de la condición testigo, siendo el tratamiento de 12 pasadas donde se registraron los mayores incrementos $(54 \%$ desde 0 a $57,5 \mathrm{~cm})$. Estos resultados demuestran que este suelo estaba en condiciones más susceptibles para compactarse a consecuencia del tráfico. A medida que se aumentó el número de pasadas, mayores diferencias se encontraron con respecto al suelo testigo. En el rango de 37,5 a 57,5 cm (capa donde el suelo testigo tenía menor resistencia a la penetración que la SD) es donde estos incrementos fueron más notorios superándose siempre el 50\% y alcanzando el 68 y $78 \%$ respectivamente para el tratamiento de 10 y 12 pasadas.

En este caso, valores absolutos de RP superiores a $2 \mathrm{MPa}$, comenzaron a registrarse a menores profundidades que para el caso del suelo con SD. Para los tratamientos de 10 y 12 pasadas desde los $32,5 \mathrm{~cm}$ y para 8 y 6 pasadas desde $10 \mathrm{c} 35 \mathrm{~cm}$. Nuevamente, los tratamientos con más número de pasadas (10 y 12) registraron valores de RP capaces de limitar el desarrollo radicular, a menores profundidades (Fig. 3).

\section{Densidad global}

Labranza convencional: Para los tres estratos de profundidad analizados, los tratamientos de menor intensidad de tráfico (6 y 8 pasadas) nunca modificaron la densidad global de la condición testigo. Por el contrario, 10 y 12 pasadas siempre la incrementaron y a su vez se diferenciaron entre ellas para las capas de 15 a $30 \mathrm{~cm}$ y 30 a $60 \mathrm{~cm}$ (Tabla 3 A).

Tabla 3. Valores de densidad global $\left(\mathrm{Mg} \mathrm{m}^{-3}\right)$ registrados con cilindros en el suelo con labranza convencional (A) y siembra directa (B) para todas las profundidades (cm) estudiadas*

\begin{tabular}{|c|c|c|c|}
\hline Prof. cm) & $0-15$ & $15-30$ & $30-60$ \\
\hline \multicolumn{4}{|c|}{ A. Labranza convencional } \\
\hline Testigo & $1,20 \mathrm{a}$ & $1,42 \mathrm{a}$ & $1,67 \mathrm{a}$ \\
\hline 6 pasadas & $1,21 \mathrm{a}$ & $1,44 \mathrm{a}$ & $1,67 \mathrm{a}$ \\
\hline 8 pasadas & $1,25 \mathrm{a}$ & 1,46 a & $1,68 \mathrm{a}$ \\
\hline 10 pasadas & $1,30 \mathrm{~b}$ & $1,50 \mathrm{~b}$ & $1,75 \mathrm{~b}$ \\
\hline 12 pasadas & $1,32 \mathrm{~b}$ & $1,59 \mathrm{c}$ & $1,84 \mathrm{c}$ \\
\hline \multicolumn{4}{|c|}{ B. Siembra directa } \\
\hline Testigo & $1,33 \mathrm{a}$ & $1,59 \mathrm{a}$ & $1,87 \mathrm{a}$ \\
\hline 6 pasadas & $1,38 \mathrm{a}$ & $1,61 \mathrm{a}$ & $1,88 \mathrm{a}$ \\
\hline 8 pasadas & $1,40 \mathrm{a}$ & $1,67 \mathrm{a}$ & $1,90 \mathrm{a}$ \\
\hline 10 pasadas & $1,51 \mathrm{~b}$ & $1,70 \mathrm{a}$ & $1,97 \mathrm{a}$ \\
\hline 12 pasadas & $1,66 \mathrm{c}$ & $1,73 \mathrm{ab}$ & $2,10 \mathrm{~b}$ \\
\hline
\end{tabular}

inicial en las columnas no tienen diferencias significativas al $5 \%$ de probabilidades del test de Tukey

En la capa más superficial analizada $(0-15 \mathrm{~cm})$ nunca se registraron valores absolutos de densidad que según la bibliografía determinarían restricciones para el desarrollo radicular de los cultivos. La capa más profunda, 30 a $60 \mathrm{~cm}$, siempre presentó valores de densidad limitantes para cualquier condición, aún el testigo. El suelo que recibió 6, 8 y 10 pasadas registró valores de densidad superiores a $1,5 \mathrm{Mg} \mathrm{m}^{-3}$ sólo en la capa más profunda; mientras que el suelo que recibió 12 pasadas, involucró también la capa entre los $15-30 \mathrm{~cm}$.

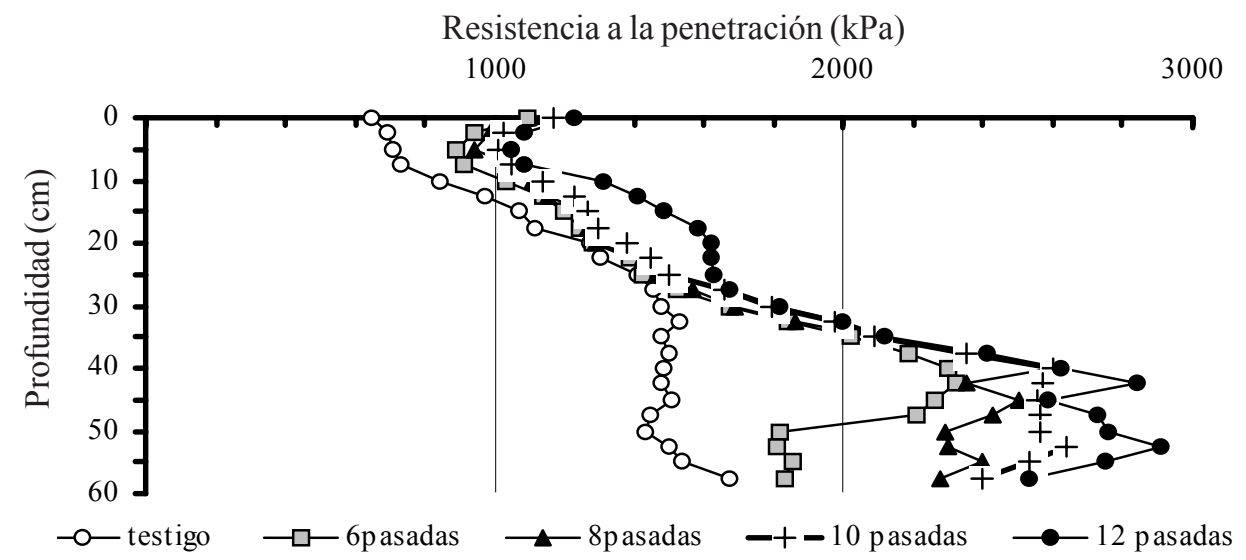

Figura 3. Resistencia a la penetración - $(\mathrm{kPa})$ según las diferentes profundidades $(\mathrm{cm})$ en el suelo con labranza convencional 
Siembra directa: Para los tres estratos de profundidad analizados, los tratamientos de 6 y 8 pasadas, nunca modificaron la densidad aparente de la condición (igual comportamiento que la LC). Las mayores intensidades de tráfico (10 y 12) la modificaron en la superficie, no en el estrato intermedio y sólo 12 pasadas en la capa más profunda (Tabla 3B).

En cuanto a los valores absolutos mayores a $1,5 \mathrm{Mg} \mathrm{m}^{-3}$, aparecen a menor profundidad que el suelo con labranza convencional, desde los $15 \mathrm{~cm}$ para todos los tratamientos y para el caso de 10 y 12 pasadas, desde la superficie.

Si se comparan estos valores con los registrados en el suelo con LC, para todas las profundidades estudiadas, los valores de densidad global de la siembra directa fueron superiores. La mayor diferencia se registró en la superficie donde el incremento alcanzó el 26\% para el tratamiento de 12 pasadas. En cuanto a los valores de resistencia a la penetración, tal como fue expuesto anteriormente, para ambos tratamientos de labranza, también es posible visualizar claras diferencias en la respuesta mecánica del suelo al tránsito, con mayores incrementos de RP a nivel superficial bajo siembra directa y en profundidad en el suelo traficado en laboreo convencional. Estos resultados se contraponen con los encontrados por Flower \& Lal (1998), quienes informaron que no existían diferencias en la distribución vertical de la compactación en diferentes sistemas de labranza.

El aumento en el número de pasadas no determinó, en forma general, que el estrato del terreno que reacciona con mayores aumentos de la resistencia a la penetración del suelo tienda a hacerse más superficial, en contraposición a lo encontrado por Jorajuría y Draghi (2000). Sin embargo, se corrobora la conclusión a la cual arriban los mismos, en lo referente a la posibilidad de inducir compactación a nivel subsuperficial con tractores de bajo peso/eje. También es posible avalar los resultados informados por Botta (2000) en cuanto a que, independientemente del peso del tractor, el tránsito reiterado produce compactación a nivel subsuperficial. La compactación del suelo no logró mantenerse, tal como encontró van den Akker (1998) dentro del horizonte arable (Ap) pese a que la carga/eje resultó inferior a $32 \mathrm{kN}$, esto podría explicarse, en parte, por el tránsito repetido en la misma huella, como también por las reducidas áreas de contacto de los rodados delanteros $\mathrm{y}$ traseros, las cuales determinaron que las presiones de contacto estimadas alcanzaran respectivament valores de 142,65 y $110,55 \mathrm{kPa}, 185,3$ y $120 \%$ mayores a la presión de inflado de $50 \mathrm{kPa}$ indicada por Alakuku (1997) cuando la humedad del terreno resulta elevada.

\section{CONCLUSIONES}

1. El suelo con siembra directa, presenta una mayor impedancia mecánica en superficie que el cultivado con labranza convencional.

2. La siembra directa registra valores de densidad global limitantes para el normal desarrollo radicular a menores intensidades de tráfico y desde menores profundidades.
3. A medida que aumenta la intensidad de tráfico disminuye la profundidad donde se alcanzan valores de resistencia a la penetración potencialmente determinantes de la detención en el crecimiento radicular.

\section{LITERATURA CITADA}

Alakukku, L. Long-term soil compaction due to high axle load traffic. Jokioinen: Agricultural Research Centre of Finland, 1997. 51p. Doctoral Thesis

ASAE Standards. 1992. S 313.4. Soil cone penetrometer. St. Joseph. 1992. 611p.

Botta, G.F. Distribución subsuperficial de la compactación inducida por el tráfico agrícola. Universidad Nacional de Luján, 2000.201p. Tesis Doctoral

Bowen H.D.; Garner, T.H.; Vaughn, D.H. Advances in soil-plant dynamics. In: ASAE. Advances in soil dynamics. St. Joseph. 1994. p.255-280.

Carter, M. Penetration resistance to characterize the depth and persistence of soil loosening tillage studies. Canadian Journal of Soil Science. Ottawa, v.68, n.4, p.657-668, 1988.

Consejo Federal de Inversiones. Eslabonamiento productivo del sector maquinaria agrícola argentina. Mercado de sembradoras presente y futuro. Buenos Aires, 2001. 84p.

Daddow, R.L.; Warrington, G.E. Growth-limiting soil bulk densities as influenced by soil texture. Fort Collins: USDA, Forest Service, 1983. p.1-17.

Dominguez, J.; Ressia, J.M.; Jorajuría, D.; Balbuena, R.; Mendivil, G. Reología del suelo bajo tres diferentes tratamientos mecánicos. In: Avances en Ingeniería Agrícola, Congreso Americano de Ingeniería Agrícola Buenos Aires. Anales: Editorial Facultad de Agronomía, 2000. p. 110-115.

Flower, M.D.; Lal, R. Axle load and tillage effects on selected soil physical properties and soybean yield on a mollic achraqualf in northwest Ohio. Soil \& Tillage Research, Amsterdam, v. 48, p. 21-35, 1998.

Ismail, I. ; Blevins, R. ; Frye, W. Long-term no-tillage effects on selected soil physical properties. Soil Science Society of America Journal, Madison, v.54, p.161-166, 1995.

Jorajuría, D. Distribución vertical de la compactación del suelo bajo tráfico vehicular agrícola. Universidad Politécnica de Valencia, España 2001.350p. Tesis Doctoral

Jorajuría, D.; Draghi, L. Sobrecompactación del suelo agrícola Parte I: Influencia del peso y del número de pasadas. Revista Brasileira de Engenharía Agrícola e Ambiental, Campina Grande, v.4, n.3, p.445-452, 2000.

Threadgill, E.D. Residual tillage effects as determined by cone index. Transactions of the ASAE, St. Joseph, v.25, n.4, p.859863,1982

van den Akker, J.J. Prevention of subsoil compaction by defining a maximum wheel load bearing capacity. In: Märländer, B.; Tijink, F.J.; Hoffman, C.; Beckers, R. (eds.) Soil compaction and compression in relation to sugar beet production: Parte 1, 1998. p.55-66. 